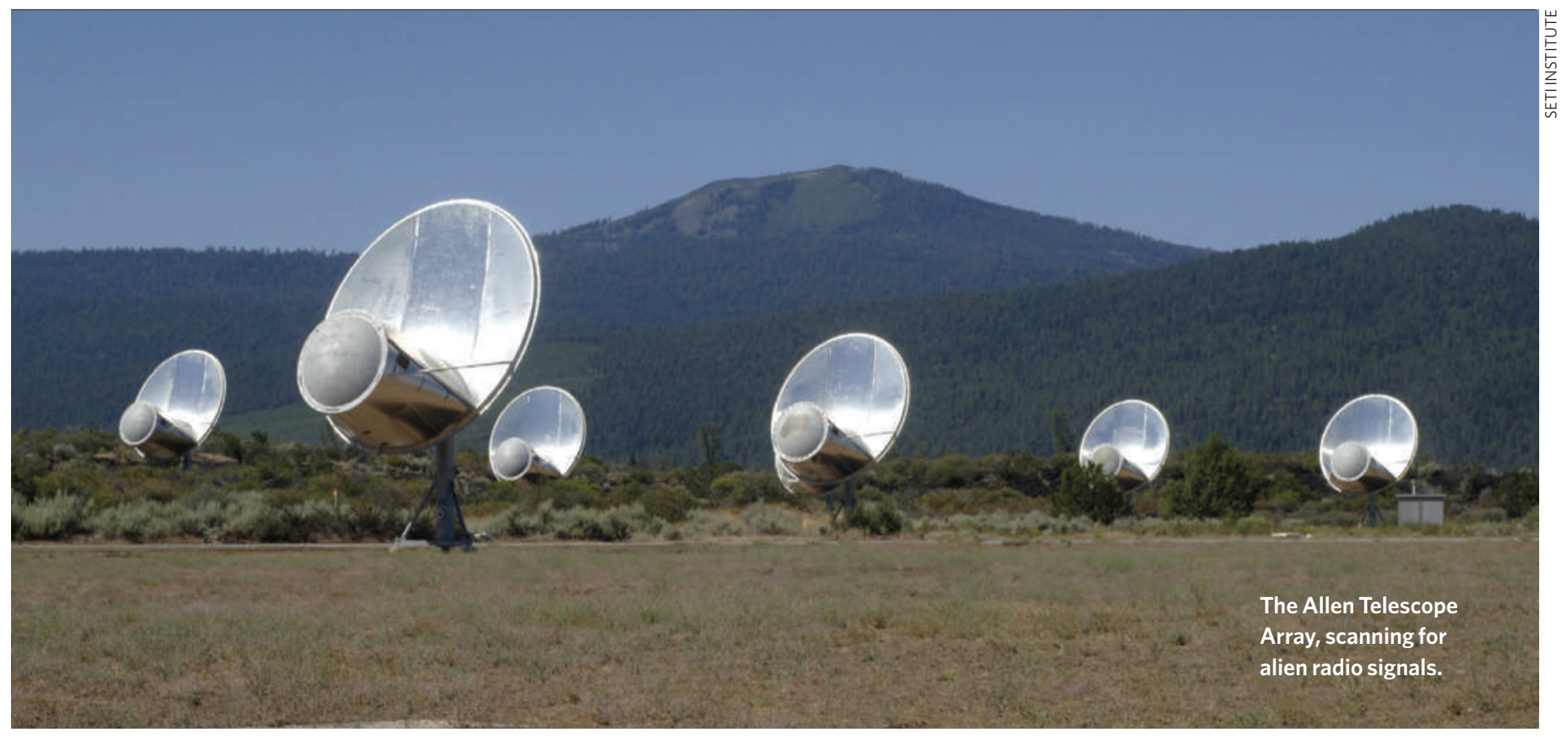

\title{
Ear to the Universe starts listening
}

A large array of radio telescopes has begun its first sustained search for extraterrestrial intelligence (SETI) and at rates faster than ever before. Even so, the project has scrambled to find money to stay open and reach its planned size. "We've had a chequered time here," says Don Backer, director of the Allen Telescope Array (ATA) in Hat Creek, California. "We're skating on thin ice."

The ATA has 42 six-metre dishes swivelling in the high desert, far fewer than the 350 dishes planned. In May, the array began combing the centre of our Milky Way Galaxy for alien signals across a broad slice of the radio spectrum. The effort comes 50 years after the concept of SETI was invented (see Opinion, page 345).

Previous searches relied on weeks-long observing runs at facilities such as the Arecibo radio telescope in Puerto Rico. The last major search, Project Phoenix — run by the SETI Institute of Mountain View, California ended in 2004 and required a decade to check 800 stars across a narrow frequency range. The ATA scans the sky much more quickly, allowing a million stars to be checked in just a few decades, says astronomer Seth Shostak of the SETI Institute, which operates the ATA jointly with the University of California at Berkeley. Shostak says sampling a million stars would offer a good chance of striking on one of the 10,000 intelligent civilizations that might be broadcasting in the Milky Way, according to an estimate by Frank Drake, who in 1960 developed a formula to estimate this number.

Private donors, often technologists, began to support SETI in 1993, after the US Congress rescinded NASA funding for it. The family foundation of Microsoft co-founder Paul Allen provided US $\$ 25$ million, beginning in 2000 , to start the ATA. But in 2006, that stream of money was cut off (see Nature 444, 9; 2006), as the SETI Institute and Berkeley struggled to find matching donations to complete the array, which to date has cost $\$ 50$ million.

Last year, the National Science Foundation (NSF) turned down a proposal to support operations at the array. SETI astronomer Jill Tarter says the NSF's decision was "like a Catch-22". The array was big enough at 42 dishes to begin work and needed money for that - but was not yet big enough to achieve the sensitivity capable of transformational science. Backer hopes that once completed, the ATA, covering vast swaths of sky rapidly, will usher in an era of transient radio astronomy - the study of things, such as supernovae, that go bump in the night rather than shine constantly like stars. Science targets could include the star-fuelling hydrogen that surrounds galaxies, and the radio afterglow of the $\gamma$-ray bursts that follow supernovae.

Without the NSF money, the \$1.5-million-ayear operation cost is being paid by the US Air
Force, which uses the array to track satellites and orbital debris. "It's keeping our doors open right now," says Backer. The Allen Foundation has given an additional \$5 million since 2006 .

Time at the array is split roughly equally: a third to the Air Force, a third to radio astronomy and third to SETI. Increasingly, however, SETI can piggyback on the radio astronomy work.

The ATA is also a testbed for technologies that will be important for the rest of radio astronomy. The array has a wide view of the sky, and within that picture, multiple stars can be analysed simultaneously. This technology, known as beam forming, as well as the immense computing challenge of making a picture from many individual dishes, will be needed in future projects, such as the Square Kilometre Array, which envisions thousands of dishes. "This is where radio astronomy has to go," says Mark McKinnon, project manager for a \$94-million expansion of the 27-dish Very Large Array in New Mexico. The ATA, he says, "are the only people who are actively doing this".

Backer has a proposal before the NSF to double the number of dishes to 84 . The request would match $\$ 6$ million in NSF money with $\$ 5$ million committed by five donors, including the Allen Foundation and Taiwan's Institute of Astronomy and Astrophysics. Backer says a decision is due before the end of the year.

Eric Hand

\section{See Editorial, page 316.}

